\title{
The XMM-Newton view of NGC 6251
}

\author{
M. Gliozzi ${ }^{1}$, R. M. Sambruna ${ }^{1}$, W. N. Brandt ${ }^{2}$, R. Mushotzky ${ }^{3}$, and M. Eracleous ${ }^{2}$ \\ 1 George Mason University, Dept. of Physics \& Astronomy \& School of Computational Sciences, 4400 University Drive, \\ MS 3F3, Fairfax, VA 22030, USA \\ 2 The Pennsylvania State University, Department of Astronomy \& Astrophysics, 525 Davey Lab, University Park, PA 16802, \\ USA \\ 3 NASA Goddard Space Flight Center, Code 662, Greenbelt, MD 20771, USA
}

Received 4 July 2003 / Accepted 20 September 2003

\begin{abstract}
We report on the nuclear X-ray properties of the radio galaxy NGC 6251 observed with XMM-Newton. NGC 6251 is a well-known radio galaxy with intermediate FRI/II radio properties. It is optically classified as a Seyfert 2 and hosts a supermassive black hole with mass $\sim 6 \times 10^{8} M_{\odot}$. The 0.4-10 keV EPIC pn continuum is best fitted by two thermal components ( $k T \sim 0.5$ and $1.4 \mathrm{keV}$, respectively), plus a power law with photon index $\Gamma \sim 1.9$ absorbed by a column density $N_{\mathrm{H}} \sim$ $5 \times 10^{20} \mathrm{~cm}^{-2}$. We confirm the previous ASCA detection of a strong iron line. The line, resolved in the EPIC pn spectrum, is adequately fitted with a broad $(\sigma \sim 0.6 \mathrm{keV})$ Gaussian at rest-frame energy $6.4 \mathrm{keV}$ with $E W \sim 220 \mathrm{eV}$. We also detect, for the first time, short-term, low-amplitude variability of the nuclear flux on a timescale of a few ks. The spectral properties argue in favor of the presence of a standard accretion disk, ruling out the base of the jet as the sole origin of the X-rays. The moderate X-ray luminosity and lack of strong intrinsic absorption suggest that NGC 6251 is a "pure" type 2 AGN which lacks a broad-line region.
\end{abstract}

Key words. galaxies: active - galaxies: nuclei - X-rays: galaxies

\section{Introduction}

NGC $6251(z=0.024)$ is a giant elliptical galaxy hosting a supermassive black hole with mass $M_{\mathrm{BH}} \sim 4-8 \times 10^{8} M_{\odot}$ (Ferrarese \& Ford 1999), as measured with HST. At radio wavelengths, the source has been well studied. It shows the typical morphology of a Fanaroff-Riley II (FR II) with a very narrow and long $\left(>4^{\prime}\right)$ jet extending N-W (e.g., Saunders et al. 1981), which emits at X-ray wavelengths (Birkinshaw \& Worrall 1993; Mack et al. 1997). However, based on its radio power at $178 \mathrm{MHz}$, NGC 6251 is classified as an FR I (e.g., Owen \& Laing 1989). Thus, NGC 6251 occupies an interesting niche in the study of radio galaxies as an intermediate object (from the radio perspective) between low- and high-power sources.

In the optical, NGC 6251 has been classified as a Seyfert 2, based on the presence of permitted and forbidden emission lines with $F W H M \sim 600 \mathrm{~km} \mathrm{~s}^{-1}$ (e.g., Shuder \& Osterbrock 1981). Previous X-ray studies of this galaxy with ROSAT showed the presence of an unresolved nuclear source embedded in diffuse thermal emission associated with the galaxy's halo (Birkinshaw \& Worrall 1993). ASCA observations showed that the nuclear spectrum could be described as a moderately absorbed power law continuum and also suggested the presence

Send offprint requests to: M. Gliozzi,

e-mail: mario@physics.gmu.edu of a strong $(E W \sim 400 \mathrm{eV})$, narrow line at $\sim 6.7 \mathrm{keV}$ (Sambruna et al. 1999; Turner et al. 1997).

Despite the intensive study of this source at all wavelengths, the nature of the accretion process in NGC 6251 is still a matter of debate. Based on the radio-to-X-ray spectral energy distribution, Ho (1999) suggested that an AdvectionDominated Accretion Flow (ADAF) is present in the nucleus of NGC 6251. On the other hand, Ferrarese \& Ford (1999) and Melia et al. (2002) favored a standard accretion disk. Similarly, the origin of the X-ray emission from the nucleus is uncertain: Turner et al. (1997) advocated a typical obscured Seyfert 2 spectrum based on the ASCA data, while Hardcastle \& Worrall (2002) argued in favor of an origin from the base of the relativistic jet for the soft X-rays. Further support for the jetdominated hypothesis was recently claimed by Chiaberge et al. (2003) based on spectral energy distribution arguments. On the other hand, Guainazzi et al. (2003) favor a scenario with two main spectral components: a blazar-like spectrum dominating the high-flux state and a Seyfert-like spectrum emerging during the low-flux state.

With its larger sensitivity in the $0.3-10 \mathrm{keV}$ band, the EPIC camera on-board XMM-Newton is an ideal instrument for investigating the origin of the $\mathrm{X}$-rays and the nature of the accretion process in NGC 6251 through timing and spectral analyses. Motivated by the above considerations, we observed NGC 6251 with XMM-Newton in AO1 for 50 ks. Here 
we report the results from the analysis of the nuclear light curve and spectrum. X-ray emission from the extended jet and halo will be discussed elsewhere (Sambruna et al. 2003). Throughout the paper we use a Friedman cosmology with $H_{0}=$ $75 \mathrm{~km} \mathrm{~s}^{-1} \mathrm{Mpc}^{-1}$ and $q_{0}=0.5$.

\section{Observations and data reduction}

We observed NGC 6251 with XMM-Newton on 2002 March 26 for a duration of $\sim 41 \mathrm{ks}$ with the EPIC pn, and for $\sim 49 \mathrm{ks}$ with EPIC MOS1 and MOS2. All of the EPIC cameras were operated in full-frame mode with a medium filter for the MOS cameras and a thin filter for the pn. The recorded events were screened to remove known hot pixels and other data flagged as bad; only data with FLAG=0 were used. The data were processed using the latest CCD gain values, and only events corresponding to pattern $0-12$ (singles, doubles, triples, and quadruples) in the MOS cameras and 0-4 (singles and doubles only, since the pn pixels are larger) in the pn camera were accepted. Arf and rmf files were created with the latest available release of the XMM-Newton Science Analysis Software (SAS 5.4). Investigation of the full-field light curves revealed the presence of a period of background flaring at the end of the observation. These events were excluded, reducing the effective total exposure time to $\sim 36 \mathrm{ks}$ for the EPIC pn and $\sim 43 \mathrm{ks}$ for the MOS cameras. The RGS data of NGC 6251 have a signal-to-noise ratio $(S / N)$ that is too low for a meaningful analysis. Background spectra and light curves were extracted from source-free regions on the same chip as the source. There are no signs of pile-up in the pn or MOS cameras according to the SAS task epatplot. With an extraction radius of $35^{\prime \prime}$ the detected count rates in the energy range $0.4-10 \mathrm{keV}$ are $(0.519 \pm 0.003) \mathrm{s}^{-1}$ for the MOS1, $(0.533 \pm 0.004) \mathrm{s}^{-1}$ for the MOS2, and $(1.810 \pm 0.007) \mathrm{s}^{-1}$ for the pn. For comparison with previous broad-band $\mathrm{X}$-ray satellites the count rates measured by the SISO on-board ASCA and the MECS onboard BeppoSAX were $\sim 0.07 \mathrm{~s}^{-1}$ and $\sim 0.06 \mathrm{~s}^{-1}$, respectively (Sambruna et al. 1999; Guainazzi et al. 2003).

Inspection of an archival Chandra observation of NGC 6251 indicates that no serendipitous sources are present within the EPIC extraction radius and that the contribution from the resolved $\mathrm{kpc}$ jet is negligible. However, no entirely reliable information on the nuclear properties of NGC 6251 can be drawn from Chandra data, due to the unfortunate location of the nucleus on the CCDs' gaps and to the pile-up.

The EPIC spectra were rebinned such that each spectral bin contains at least 100 counts for the pn and 40 counts for the MOS cameras in order to apply $\chi^{2}$ minimization, and fitted jointly using the XSPEC v 11.2 software package (Arnaud 1996). The quoted errors on the derived best-fitting model parameters correspond to a $90 \%$ confidence level for one parameter of interest (i.e., a $\Delta \chi^{2}=2.7$ criterion) unless otherwise stated.

\section{EPIC light curve of the nucleus}

We first study the $0.3-10 \mathrm{keV}$ light curve obtained with the EPIC pn, which is the most sensitive instrument on-board

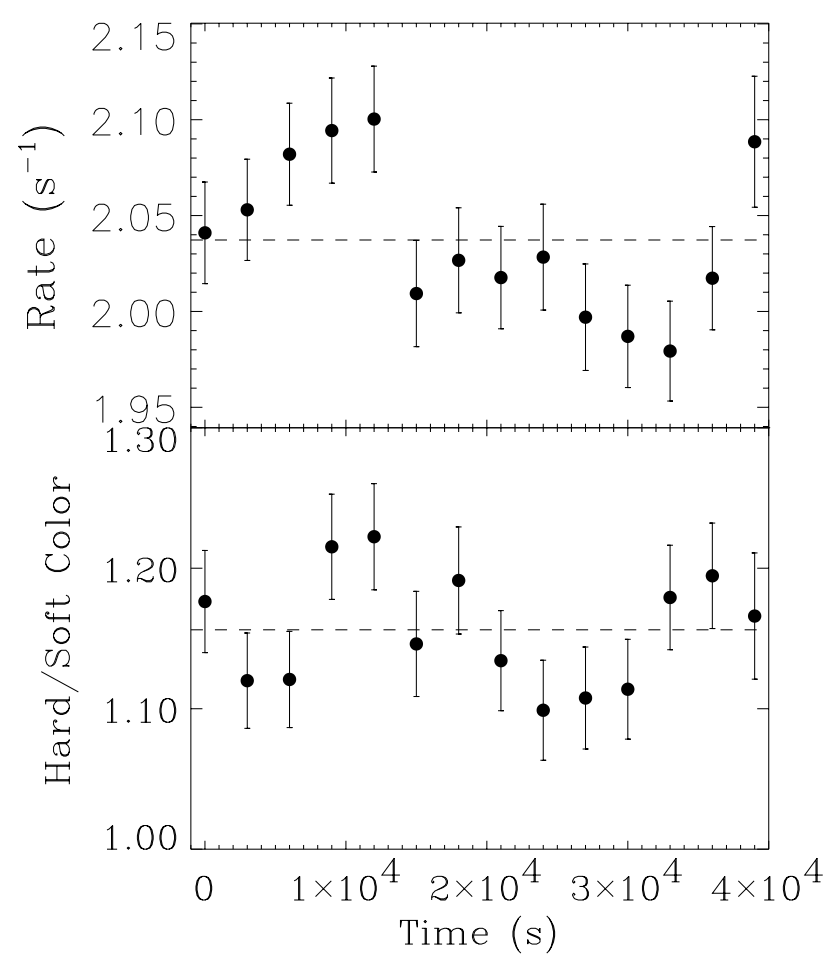

Fig. 1. EPIC pn+MOS1+MOS2 light curves of the backgroundsubtracted count rate in the $0.8-10 \mathrm{keV}$ band (top panel) and of the X-ray color $1.8-10 \mathrm{keV} / 0.8-1.5 \mathrm{keV}$ (bottom panel). The extraction radius is $35^{\prime \prime}$; time bins are $3000 \mathrm{~s}$. The dashed lines indicate the average values.

$X M M$-Newton, using an extraction radius of $35^{\prime \prime}$ and a time bin of $3000 \mathrm{~s}$ (changing the extraction radius or the time bin has a negligible impact on our results). At energies below $0.8 \mathrm{keV}$ no statistically significant variability is detected according to a $\chi^{2}$ test. This might be partly due to a combination of dilution from the thermal emission and intrinsic absorption. However, the small intrinsic absorption and the relatively small contribution of the thermal component in the low energy range (see Sect. 4) suggest that the very-soft $(0.4-0.7 \mathrm{keV})$ flux might be intrinsically constant or, at most, variable at a very low level. We therefore focus on the $0.8-10 \mathrm{keV}$ energy band. The combination of pn, MOS1, and MOS2 light curves increases the statistical significance of the variability. Figure 1 (top panel) shows the $0.8-10 \mathrm{keV}$ backgroundsubtracted EPIC pn+MOS1+MOS2 light curve of NGC 6251. The bottom panel shows a plot of the hardness ratio, defined as the ratio of the $1.8-10 \mathrm{keV}$ count rate to the $0.8-1.5 \mathrm{keV}$ count rate, versus time. Low-amplitude flux variability is present on timescales of a few ks. A $\chi^{2}$ test yields a probability that the count rate is constant of $P_{\chi^{2}}=0.9 \%$. The background count rate is as low as $5 \%$ of the average source count rate. The variability of the hardness ratio is not significant $\left(P_{\chi^{2}} \sim 22 \%\right)$.

The study of energy-dependent variability is hampered by the presence of photons emitted from the diffuse component that dilutes the intrinsic variability of the source, especially at low energies. Therefore, we have 1) divided the $0.8-10 \mathrm{keV}$ range into several sub-bands; 2) checked with a $\chi^{2}$ test the presence of variability in each sub-band; 3 ) merged contiguous variable sub-bands, creating four final bands with roughly 
Table 1. Short-term X-ray variability of NGC 6251.

\begin{tabular}{ccccc}
\hline \hline $\begin{array}{c}\text { Energy band } \\
(\mathrm{keV})\end{array}$ & $\begin{array}{c}\langle r\rangle \\
\left(\mathrm{s}^{-1}\right)\end{array}$ & $\begin{array}{c}\chi_{\text {red }}^{2} \\
(14 \text { d.o.f. })\end{array}$ & $\begin{array}{c}P_{\chi^{2}} \\
(\%)\end{array}$ & $\begin{array}{c}F_{\text {var }}{ }^{a} \\
(\%)\end{array}$ \\
\hline $0.4-0.7 \mathrm{keV}$ & 0.49 & 0.54 & 89.6 & - \\
$0.8-1 \mathrm{keV}$ & 0.34 & 1.40 & 14.8 & $2.2 \pm 1.4$ \\
$1.2-1.5 \mathrm{keV}$ & 0.36 & 1.48 & 11.7 & $2.1 \pm 1.3$ \\
$1.8-3 \mathrm{keV}$ & 0.41 & 1.59 & 8.1 & $2.3 \pm 1.2$ \\
$3-10 \mathrm{keV}$ & 0.39 & 1.66 & 6.2 & $2.8 \pm 1.3$ \\
\hline
\end{tabular}

${ }^{a}$ The errors on $F_{\text {var }}$ are calculated as in Edelson et al. (2002) and should be considered conservative estimates of the true uncertainty.

the same count rates. The energy-dependent variability has been characterized by means of the $\chi^{2}$ chance probability and the fractional variability parameter, $F_{\text {var }}=\left(\sigma^{2}-\Delta^{2}\right)^{1 / 2} /\langle r\rangle$, where $\sigma^{2}$ is the variance, $\langle r\rangle$ the unweighted mean count rate, and $\Delta^{2}$ the mean square value of the uncertainties associated with each individual count rate. The results are reported in Table 1 . For comparison, the $0.4-0.7 \mathrm{keV}$ results are also reported. No clear variability trend with energy is found.

NGC 6251 has been observed by several X-ray satellites over the last decade. Therefore, its long-term temporal behavior can be investigated. A comparison of the $2-10 \mathrm{keV}$ absorbed flux detected by ASCA in October 1994 (Sambruna et al. 1999), $F_{\mathrm{ASCA}} \sim 1.4 \times 10^{-12} \mathrm{erg} \mathrm{cm}^{-2} \mathrm{~s}^{-1}$, with values more recently measured with BeppoSAX (observation carried out in July 2001), $F_{\mathrm{SAX}} \sim 4.7 \times 10^{-12} \mathrm{erg} \mathrm{cm}^{-2} \mathrm{~s}^{-1}$, and with $X M M-N e w t o n$ (see below) $F_{\text {XMM }} \sim 4 \times 10^{-12} \mathrm{erg} \mathrm{cm}^{-2} \mathrm{~s}^{-1}$, suggests an increase by a factor of three over the last nine years. Although this conclusion might be weakened by systematic errors related to the instrumental cross-calibration uncertainties, the spectral uncertainties, and the different extraction areas, the substantial flux difference measured argues in favor of genuine variability. Observations with the same satellite are needed to carefully investigate the long-term variability.

\section{The nuclear spectrum}

\subsection{The continuum}

Motivated by the controversial results of previous X-ray spectral studies, we fitted the EPIC spectra in the $0.4-10 \mathrm{keV}$ range, where the instruments are best calibrated, with models of increasing complexity. To account for residual calibration problems between the EPIC cameras, we first fitted separately the pn, MOS1, and MOS2 spectra. All the spectral models assume a column density, obscuring all components, fixed at the Galactic value of $N_{\mathrm{H}}=5.65 \times 10^{20} \mathrm{~cm}^{-2}$ (Dickey \& Lockman 1990). In some models an additional intrinsic absorber at the redshift of NGC 6251 is included. A power-law model modified by Galactic absorption gives unacceptable fits: $\chi^{2} /$ d.o.f. are $927.8 / 443,401.2 / 232,384.5 / 233$, for the pn, MOS1, and MOS2, respectively. A first significant improvement $\left(\Delta \chi^{2} \gtrsim 80\right.$ for three additional parameters) is obtained by adding a soft component modeled as emission by a collisionally-ionized plasma (apec in XSPEC; Smith et al. 2001). However, a fair representation of the EPIC spectra is only obtained with a thermal component plus an absorbed



Fig. 2. EPIC pn unfolded spectrum of NGC 6251 described by a thermal component plus an absorbed power law. The lower panels show the data-to-model ratio for the pn, MOS1, and MOS2 cameras, respectively. The edge at $0.53 \mathrm{keV}$ in the top panel is due to neutral oxygen in the absorbing material.

power law. The column density of cold gas toward the nucleus $N_{\mathrm{H}} \simeq 5 \times 10^{20} \mathrm{~cm}^{-2}$, although small, is statistically highly significant: $\Delta \chi^{2} \sim 70-100$ for one additional parameter. We have investigated the possibility that the intrinsic absorber is ionized (using the absori in XSPEC) or partially covering the nuclear source (using the zpcfabs model in XSPEC). However, neither of these models resulted in a significant improvement of the fit. The best-fit parameters for the model consisting of a thermal component plus an absorbed power law, are reported in Table 2a (the abundances were frozen at the best-fit values). Figure 2 shows the unfolded spectrum of the EPIC pn and the data-to-model ratio for the three EPIC cameras. A close inspection of residuals shows a more complex pattern in the pn data. Indeed the pn fit is further improved by adding a second thermal component. This hotter thermal component is consistent with the one detected in the circumnuclear gas around NGC 6251 (Sambruna et al. 2003). On the other hand, the fits to the MOS spectra are not improved significantly because of their lower $S / N$. We also tried an alternative model for the pn continuum with two power laws plus a thermal component. However, the resulting spectral fit, $\left(k T=0.59_{-0.05}^{+0.06} \mathrm{keV}, Z=0.18 Z_{\odot}\right.$, 
Table 2. Spectral model parameters for NGC 6251. Parameters without errors have been fixed at the best-fit values.

\begin{tabular}{|c|c|c|c|c|c|c|c|c|c|}
\hline \multicolumn{10}{|c|}{ (a) EPIC continuum parameters } \\
\hline \multicolumn{4}{|c|}{ thermal } & \multicolumn{3}{|c|}{ absorbed PL } & \multirow{3}{*}{$\chi^{2} /$ d.o.f. } & \multirow{3}{*}{$\begin{array}{c}\text { Count rate } \\
\mathrm{s}^{-1} \\
\end{array}$} & \\
\hline Camera & $k T$ & $Z$ & norm $_{\text {apec }}$ & $N_{\mathrm{H}}$ & $\bar{\Gamma}$ & norm $_{\text {pow }}$ & & & \\
\hline & $\mathrm{keV}$ & $Z_{\odot}$ & $10^{-4} \mathrm{~cm}^{-5}$ & $10^{20} \mathrm{~cm}^{-2}$ & & $10^{-3} F_{1 \mathrm{keV}}^{i}$ & & & \\
\hline MOS1 & $0.59_{-0.08}^{+0.09}$ & 0.19 & $3.03_{-0.82}^{+0.91}$ & $6.5_{-0.9}^{+1.4}$ & $1.86_{-0.04}^{+0.04}$ & $1.19_{-0.04}^{+0.05}$ & $243.8 / 229$ & $0.519 \pm 0.003$ & \\
\hline MOS2 & $0.52_{-0.11}^{+0.12}$ & 0.18 & $3.03_{-1.45}^{+1.07}$ & $7.3_{-1.8}^{+0.7}$ & $1.92_{-0.05}^{+0.03}$ & $1.31_{-0.06}^{+0.03}$ & $236.0 / 230$ & $0.533 \pm 0.004$ & \\
\hline $\mathrm{PN}$ & $0.60_{-0.05}^{+0.06}$ & 0.18 & $3.30_{-0.48}^{+0.76}$ & $5.0_{-0.7}^{+0.5}$ & $1.91_{-0.02}^{+0.03}$ & $1.38_{-0.05}^{+0.02}$ & $548.4 / 440$ & $1.810 \pm 0.007$ & \\
\hline \multirow{2}{*}{\multicolumn{10}{|c|}{ b) Best-fitting continuum: EPIC pn }} \\
\hline & & & & & & & & & \\
\hline$k T_{1}$ & $Z_{1}$ & norm $_{\text {apec }_{1}}$ & $k T_{2}$ & $Z_{2}$ & norm $_{\text {apec }_{2}}$ & $N_{\mathrm{H}}$ & $\Gamma$ & norm $_{\text {pow }}$ & $\chi^{2} /$ d.o.f. \\
\hline $\mathrm{keV}$ & $Z_{\odot}$ & $10^{-4} \mathrm{~cm}^{-5}$ & $\mathrm{keV}$ & $Z_{\odot}$ & $10^{-4} \mathrm{~cm}^{-5}$ & $10^{20} \mathrm{~cm}^{-2}$ & & $10^{-3} F_{1 \mathrm{keV}}^{i}$ & $\mathrm{~s}^{-1}$ \\
\hline $1.36_{-0.20}^{+0.34}$ & 0.31 & $2.25_{-1.05}^{+1.37}$ & $0.54_{-0.08}^{+0.06}$ & 0.17 & $3.23_{-0.77}^{+0.72}$ & $5.1_{-0.9}^{+1.1}$ & $1.91_{-0.05}^{+0.08}$ & $1.91_{-0.05}^{+0.08}$ & $520.3 / 435$ \\
\hline \multicolumn{10}{|c|}{ c) Line parameters: EPIC pn } \\
\hline & & & $E$ & $\sigma$ & flux & $E W$ & & & \\
\hline & & & $\mathrm{keV}$ & $\mathrm{keV}$ & $10^{-6} \mathrm{~s}^{-1} \mathrm{~cm}^{-2}$ & $\mathrm{eV}$ & & & \\
\hline & & & $6.42_{-0.41}^{+0.52}$ & $0.58_{-0.24}^{+0.93}$ & $8.5_{-3.8}^{+8.3}$ & $223_{-99}^{+219}$ & & & \\
\hline
\end{tabular}

${ }^{i}$ photon $\mathrm{keV}^{-1} \mathrm{~cm}^{-2} \mathrm{~s}^{-1}$.

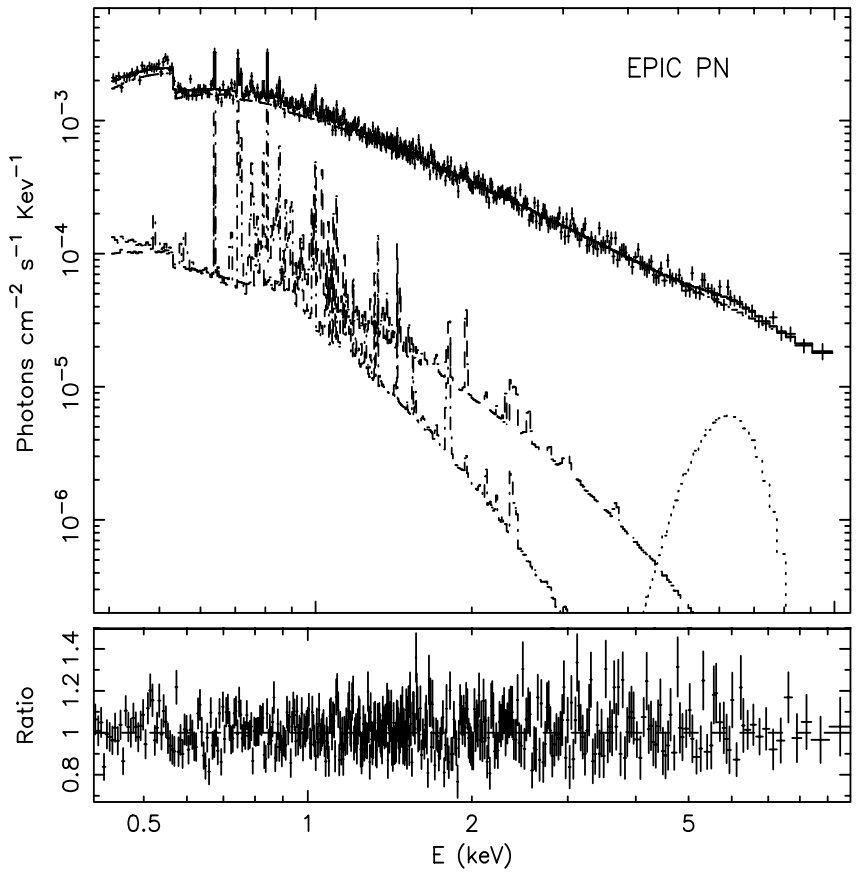

Fig. 3. EPIC pn unfolded spectrum of NGC 6251 and datato-model ratio. The spectral model is wabs(apec+apec+zwabs (powerlaw+zgauss)).

$\left.\Gamma_{1}=2.68_{-0.33}^{+0.54}, \Gamma_{2}=1.63_{-0.03}^{+0.02}\right)$ is statistically worse than the one obtained with two thermal models and one absorbed power law $\left(\Delta \chi^{2}=+9.6\right.$ for the same d.o.f.).

In addition, the residuals of the pn and MOS2 data show a clear excess in the 6-7 keV range, suggesting the presence of a line (see Sect. 4.2). The best-fitting continuum parameters are summarized in Table $2 \mathrm{~b}$ and the unfolded spectrum plus data-to-model ratio is shown in Fig. 3.
The total absorbed fluxes from $0.4-2$ and $2-10 \mathrm{keV}$ are $\sim 2.6 \times 10^{-12} \mathrm{erg} \mathrm{cm}^{-2} \mathrm{~s}^{-1}$ and $\sim 4.0 \times 10^{-12} \mathrm{erg} \mathrm{cm}^{-2} \mathrm{~s}^{-1}$, respectively, corresponding to intrinsic (absorption-corrected) luminosities of $L_{0.4-2 \mathrm{keV}} \sim 4.4 \times 10^{42} \mathrm{erg} \mathrm{s}^{-1}$ and $L_{2-10 \mathrm{keV}} \sim$ $4.8 \times 10^{42} \mathrm{erg} \mathrm{s}^{-1}$. The contribution of the thermal component is $\sim 10 \%$ from $0.4-2 \mathrm{keV}$ and negligible above $2 \mathrm{keV}$. This leads to an X-ray luminosity associated with the power-law component of $L_{0.4-10 \mathrm{keV}}^{\mathrm{PL}} \sim 8.8 \times 10^{42} \mathrm{erg} \mathrm{s}^{-1}$. This value can be further increased up to $1.7 \times 10^{43} \mathrm{erg} \mathrm{s}^{-1}$ by extending the energy range up to $100 \mathrm{keV}$. In fact, analysis of a BeppoSAX observation of NGC 6251 shows that the PDS detects the source up to energies higher than $100 \mathrm{keV}$ (Guainazzi et al. 2003).

\subsection{The Fe line}

To investigate the possible presence of an $\mathrm{Fe} \mathrm{K} \alpha$ line, we used only pn data. In the MOS cameras, the rapid decrease of quantum efficiency with increasing energy reduces the $S / N$ above $6 \mathrm{keV}$, hampering a thorough analysis of the $\mathrm{Fe} \mathrm{K} \alpha$ range. To reduce the influence of the complex continuum modeling on the line detection and properties, we restrict the energy range to $2-10 \mathrm{keV}$. The $5-7.5 \mathrm{keV}$ band was initially excluded to allow a better determination of the photon index. A simple power law model produces a satisfactory fit to the the 2 $5 \mathrm{keV}$ and 7.5-10 keV windows of the pn spectrum, with $\chi^{2}=$ 134.1 for 123 degrees of freedom. Including the $5-7.5 \mathrm{keV}$ energy range, the fit is still formally acceptable $\left(\chi^{2}=173.1\right.$, 158 d.o.f.). However, residuals around 6-7 keV (Fig. 4 top panel) suggest the presence of a broad line. Indeed, adding a Gaussian line improves the fit significantly $\left(\Delta \chi^{2}=14\right.$ for 3 additional parameters), as can be seen from the residuals in the bottom panel of Fig. 4. For testing purposes, we applied the same procedure to MOS2 data. A marginally significant $\left(\Delta \chi^{2}=7.1\right.$ for three additional parameters) line at $\sim 6.4 \mathrm{keV}$ is detected, with parameters consistent within the errors with 


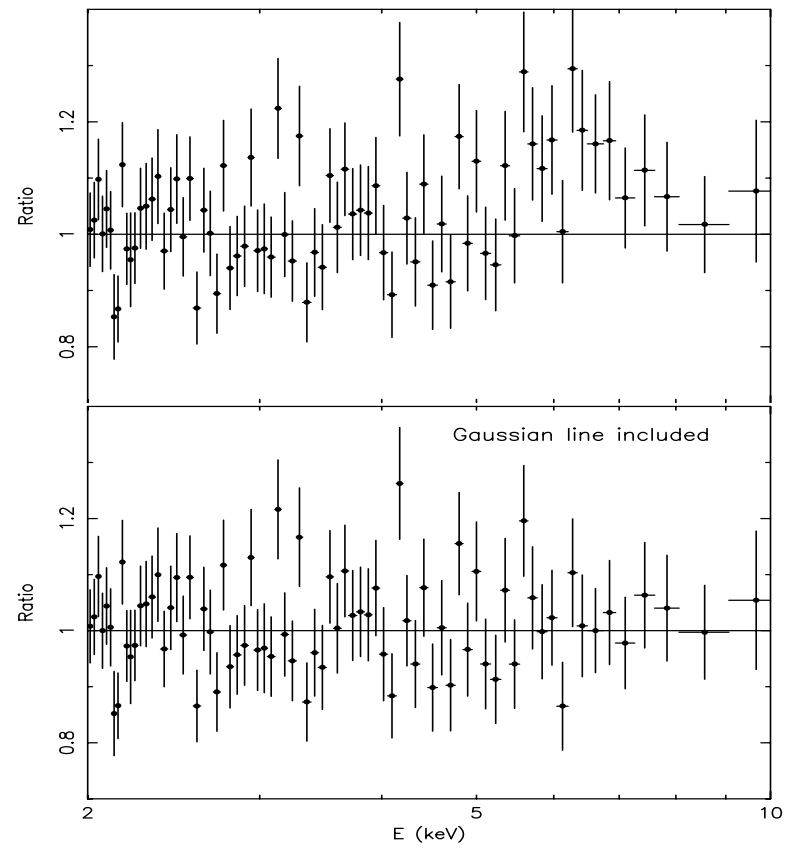

Fig. 4. Top panel: EPIC pn data-to-model ratio from $2-10 \mathrm{keV}$ of the best-fit continuum model for the EPIC pn data. Bottom panel: same as above but with a Gaussian line included. Energies are in the observer's frame.

the ones inferred using pn data. Using the entire $0.4-10 \mathrm{keV}$ range and the best-fitting model, the statistical significance of the line detection is further enhanced: $\Delta \chi^{2} \simeq 15.5$ for both $\mathrm{pn}$ and MOS2.

We also tried more complex fits to the broad line using specific disk models in XSPEC, such as diskline and laor. The former model parameterizes the expected line profile from a disk around a Schwarzschild black hole (Fabian et al. 1989), whereas the latter describes the line profile in the case of a rotating Kerr black hole (Laor 1991). In both cases, the fitted inner radius of the disk is located within a few Schwarzschild radii from the black hole; however, due to the limited $S / N$, the line parameters are poorly constrained.

In summary, we find evidence for a broad line at rest-frame energy $\sim 6.4 \mathrm{keV}$ with $E W \simeq 220 \mathrm{eV}$ and width $F W H M \simeq$ $64000 \mathrm{~km} \mathrm{~s}^{-1}$, which we interpret as the fluorescent Fe K $\alpha$ line commonly observed in Seyfert galaxies. The line parameters with the $90 \%$ confidence errors are given in Table $2 \mathrm{c}$.

\section{Discussion and conclusions}

A detailed discussion of the origin of the X-rays from weak radio galaxies has been reported by Gliozzi et al. (2003) for the specific case of NGC 4261. For that object, a jet origin, claimed on the basis of a tight correlation observed between radio and X-ray core luminosities, was disputed on the basis of variability properties and energetic considerations. On the other hand, for NGC 6251 rapid variability is detected but no clear flux or spectral variability trend is found, and the investigation of the origin of the X-rays must rely upon the time-averaged spectral results.

The strongest argument against a jet origin comes from the detection of a prominent broad $\mathrm{Fe} \mathrm{K} \alpha$ line. The large $E W$ is consistent with the average value found in Seyfert 1 galaxies (e.g., Nandra et al. 1997) and with the upper limits found in brighter radio-loud AGN (e.g., Sambruna et al. 1999), and is much larger than typical values found in broad-line radio galaxies observed with RXTE (e.g., Eracleous et al. 2000), arguing against a significant contribution from the jet. A Dopplerbroadened line with $F W H M \simeq 64000 \mathrm{~km} \mathrm{~s}^{-1}$ can only be produced in the inner region of an accretion flow by fluorescence (although a deeper X-ray exposure is necessary to constrain the inner radius of the line-emitting region). Contributions from the BLR are excluded here because the source lacks optical broad lines (e.g., Shuder \& Osterbrock 1981). Alternative scenarios invoking a jet with a wide opening angle illuminating the putative obscuring torus can only produce narrow emission lines (Woźniak et al. 1998).

In addition, the well constrained photon index, $\Gamma \simeq 1.9$, agrees very well with the average value found in Seyfert 1 galaxies (e.g., Nandra et al. 1997); however, it is not inconsistent with photon indices derived for X-ray jets of FR I radio galaxies, even though the latter are generally poorly constrained (e.g., Hardcastle et al. 2002).

The small absorbing column is in good agreement with the low visual extinction $A_{\mathrm{V}} \sim 0.6$ inferred for the dusty disk (Ferrarese \& Ford 1999), assuming the Galactic gas-to-dust ratio. The lack of strong intrinsic absorption in NGC 6251 argues against obscuration by a canonical molecular torus (see, e.g., Chiaberge et al. 2002). The issue of intrinsic absorption in FR I galaxies will be addressed in a forthcoming paper (Donato et al. 2003, in prep).

Assuming that (most of) the X-rays are not associated with the jet, we investigate the nature of the accretion flow. The main question is whether an ADAF or a standard disk model provides a better description of the accretion process in NGC 6251. To answer this question, we first note that the ADAF interpretation proposed by Ho (1999) was mainly prompted by the low value inferred for the ratio $L_{\mathrm{bol}} / L_{\mathrm{Edd}}$, where $L_{\mathrm{bol}}=$ $8.2 \times 10^{42} \mathrm{erg} \mathrm{s}^{-1}$ was obtained by integrating the spectral energy distribution from radio to X-rays. However, because of the paucity of data points, the estimate of $L_{\mathrm{bol}}$ is highly uncertain. Indeed, Ferrarese \& Ford (1999) and Melia et al. (2002) independently derive a value of $L_{\text {bol }}$ larger by a factor of a few with respect to the value derived by Ho. Direct evidence that the value derived by Ho underestimates $L_{\mathrm{bol}}$ comes from the value of X-ray luminosity $L_{0.4-10 \mathrm{keV}}^{\mathrm{PL}} \sim 8.8 \times 10^{42} \mathrm{erg} \mathrm{s}^{-1}$ associated with the power-law component. Therefore, assuming that $L_{0.4-10 \mathrm{keV}}^{\mathrm{PL}}$ represents $\sim 30 \%$ of $L_{\mathrm{bol}}$ (see, e.g., Elvis et al. 1994), we obtain a more reasonable but still conservative estimate of $L_{\text {bol }}$ of the order of $3 \times 10^{43} \mathrm{erg} \mathrm{s}^{-1}$, consistent with the estimate given by Ferrarese \& Ford (1999). However, the modest value of the ratio $L_{\mathrm{bol}} / L_{\mathrm{Edd}}$ derived from this assumption does not allow us to discriminate between the competing accretion scenarios.

An independent argument favoring a standard accretion disk is the detection of a broad $\mathrm{Fe} \mathrm{K} \alpha$ line. As already mentioned, the most natural explanation for the broad line detected at $\sim 6.4 \mathrm{keV}$ is that it is a fluorescent $\mathrm{Fe} \mathrm{K} \alpha$ line produced within the inner gravitational radii of a optically thick accretion disk. 
Direct evidence against radiatively inefficient solutions comes from the estimate of the radiative efficiency $\eta$, which can be derived comparing the accretion luminosity $L_{\text {accr }}=$ $\eta \dot{M}_{\text {accr }} c^{2}$ to $L_{\mathrm{bol}}$. A rough estimate of the accretion rate is given by the Bondi value, $\dot{M}_{\text {Bondi }}=4 \pi R_{\mathrm{A}}^{2} \rho_{\mathrm{A}} c_{\mathrm{s}}$ where $R_{\mathrm{A}} \simeq G M / c_{\mathrm{s}}^{2}$ is the accretion radius, $c_{\mathrm{s}}$ the sound speed, and $\rho_{\mathrm{A}}$ the density at the accretion radius. Since the soft thermal component $(k T \sim 0.55 \mathrm{keV})$ is spatially unresolved with XMM-Newton (Sambruna et al. 2003), it is appropriate to choose the instrument resolution, 10", as the angular size of the thermal component. With this assumption, and considering normal cosmic composition $\left(n_{\mathrm{e}} / n_{\mathrm{p}}=1.18\right)$, the emission measure given by the normalization of the soft thermal component, $\left[10^{-14} /\left(4 \pi\left(D_{\mathrm{A}}(1+z)\right)^{2}\right)\right] \int n_{\mathrm{e}} n_{\mathrm{H}} \mathrm{d} V \simeq 3 \times 10^{-4} \mathrm{~cm}^{-5}$ (where $D_{\mathrm{A}}$ is the angular size distance to the source in $\mathrm{cm}$, and $n_{\mathrm{e}}$ and $n_{\mathrm{H}}$ are the electron and $\mathrm{H}$ densities in $\mathrm{cm}^{-3}$ ) yields a proton density of $n_{\mathrm{p}} \simeq 0.06 \mathrm{~cm}^{-3}$, which translates into $\dot{M}_{\text {Bondi }} \simeq 8 \times 10^{-3} M_{\odot} \mathrm{yr}^{-1}$. A direct comparison of this value with the estimated $L_{\text {bol }}$ leads to a radiative efficiency of the order of $\eta \sim 0.06$, fully consistent with the standard disk scenario. This conclusion is supported by the striking difference observed between NGC 6251, that radiates at the Bondi limit, and low luminosity AGN observed with Chandra (Loewenstein et al. 2001) which show $L_{\text {bol }} \ll L_{\text {Bondi }}$ and are interpreted in the ADAF framework.

A final important issue to be addressed is the nature of the Seyfert nucleus in NGC 6251. Even though historically this source has been classified as a type 2 AGN, its X-ray properties as seen with $X M M$-Newton seem to be more typical for a type 1 AGN. Interestingly, we note that the value inferred for $L_{\text {bol }} / L_{\text {Edd }}$ translates into $\dot{M} / \dot{M}_{\text {Edd }}$ in the range where recent models (e.g., Nicastro 2000; Laor 2003) predict that a broad-line region (BLR) cannot exist. The absence of broad optical lines and the low value inferred for the cold absorption from the X-ray spectral analysis support the hypothesis that NGC 6251 is a "pure" type 2 AGN, i.e., without BLR. This result is consistent with the findings of Steffen et al. (2003), who, studying sources of the X-ray background, find that the fraction of objects with broad lines drops sharply below $L_{\mathrm{X}} \sim 10^{44} \mathrm{erg} \mathrm{s}^{-1}$. An alternative interpretation might be that the source is Compton-thick and the observed X-rays are only the scattered component. A possible way to test this hypothesis is based on the location of NGC 6251 in the Fe $E W-$ $L_{\mathrm{X}} / L_{\mathrm{OIII}}$ plane (Bassani et al. 1999). However, such analysis does not provide any firm conclusion, since NGC 6251 is located half way between the Compton thick region and the Seyfert 1 region. The rapid X-ray variability, the rather weak iron line for a type $2 \mathrm{AGN}$, and the BeppoSAX results (Guainazzi et al. 2003) seem to argue against the Comptonthick hypothesis. However, deep exposures at hard X-rays are necessary to discriminate between the two competing scenarios.

A recent cross-correlation study of the FIRST and 2DF catalogs (Magliocchetti et al. 2002) finds that the majority of radio sources do not show any optical emission lines, while a minority have spectra similar to NGC 6251. If NGC 6251 with its Seyfert 1-like nuclear properties is representative of the whole group, it follows that there is a significant fraction of sources which do not fit the standard view of AGN. Detailed multiwavelength studies of their nuclear properties are needed.

Acknowledgements. We thank the anonymous referee for the useful comments and suggestions that improved the paper. Financial support from NASA LTSA grants NAG5-10708 (MG, RMS), NAG5-13035 (WNB), and NAG5-10817 (ME) is gratefully acknowledged. Funds were also provided by NASA grant NAG5-10243 (MG, RMS) and by the Clare Boothe Luce Program of the Henry Luce Foundation (RMS).

\section{References}

Arnaud, K. 1996, in Astronomical Data Analysis Software and Systems V, ed. G. Jacoby, \& J. Barnes (San Francisco: ASP), ASP Conf. Ser., 101, 17

Bassani, L., Dadina, M., Maiolino, R., et al. 1999, ApJS, 121, 473

Birkinshaw, M., \& Worrall, D. M. 1993, ApJ, 412, 568

Chiaberge, M., Macchetto, F. D., Sparks, W. B., et al. 2002, ApJ, 571, 247

Chiaberge, M., Gilli, R., Capetti, A., \& Macchetto, F. D. 2003, ApJ, 597, 166

Dickey, J. M., \& Lockman, F. J. 1990, ARA\&A, 28, 215

Edelson, R., Turner, T. J., \& Pounds, K. 2002, ApJ, 568, 610

Elvis, M., Wilkes, B. J., McDowell, J. C., et al. 1994, ApJS, 95, 1

Eracleous, M., Sambruna, R. M., \& Mushotzky, R. F. 2000, ApJ, 537, 654

Fabian, A. C., Rees, M. J., Stella, L., \& White, N. E. 1989, MNRAS, 238,729

Ferrarese, L., \& Ford, H. C. 1999, ApJ, 515, 583

Gliozzi, M., Sambruna, R. M., \& Brandt, W. N. 2003, A\&A, 408, 949

Guainazzi, M., Grandi, P., Comastri, A., \& Matt, G. 2003, A\&A, 410, 131

Hardcastle, M. J., \& Worrall, D. M. 2000, MNRAS, 314, 359

Hardcastle, M. J., Worrall, D. M., Birkinshaw, M., Laing, R. A., \& Bridle, A. H. 2002, MNRAS, 334, 182

Ho, L. C. 1999, ApJ, 516, 672

Laor, A. 1991, ApJ, 376, 90

Laor, A. 2003, ApJ, 590, 86

Loewenstein, M., Mushotzky, R. F., Angelini, L., Arnaud, K. A., \& Quataert, E. 2001, ApJ, 555, L21

Mack, K.-H., Kerp, J., \& Klein, U. 1997, A\&A, 324, 870

Melia, F., Liu, S., \& Fatuzzo, M. 2002, ApJ, 567, 811

Magliocchetti, M., Maddox, S. J., Jackson, C. A., et al. 2002, MNRAS, 333, 100

Owen, F. N., \& Laing, R. A. 1989, MNRAS, 238, 357

Nandra, K., George, I. M., Mushotzky, R. F., Turner, T. J., \& Yaqoob, T. 1997, ApJ, 477, 602

Nicastro, F. 2000, ApJ, 530, L65

Sambruna, R. M., Eracleous, M., \& Mushotzky, R. 1999, ApJ, 526, 60

Sambruna, R. M., et al. 2003, A\&A, submitted

Saunders, R., Baldwin, J. E., Pooley, G. G., \& Warner, P. J. 1981, MNRAS, 197, 287

Smith, R. K., Brickhouse, N. S., Liedahl, D. A., \& Raymond, J. C. 2001, ApJ, 556, L91

Shuder, J. M., \& Osterbrock, D. E. 1981, ApJ, 250, 55

Steffen, A. T., Barger, A. J., Cowie, L. L., Mushotzky, R., \& Yang, Y. 2003, ApJ, 596, L23

Turner, T. J., George, I. M., Nandra, K., \& Mushotzky, R. F. 1997, ApJS, 113, 23

Woźniak, P. R., Zdziarski, A. A., Smith, D., Madejski, G. M., \& Johnson, W. N. 1998, MNRAS, 299, 449 